\title{
DNA Base Composition and Taxonomy of Phytopathogenic and Other Enterobacteria
}

\author{
By M. P. STARR \\ Department of Bacteriology, University of California, Davis, \\ California 95616, U.S.A. \\ AND M. MANDEL \\ Department of Biology, University of Texas M.D. Anderson \\ Hospital and Tumor Institute at Houston, Houston, Texas 77025, U.S.A.
}

(Accepted for publication 6 December I968)

SUMMARY

Using lysates and purified DNA, base ratios were determined by the buoyant density ultracentrifugation and the absorbance-temperature transition techniques; the correspondence was very high between the two methods. The DNAs from a large number of Erwinia species, mostly phytopathogenic and nonphytopathogenic, but including some 'Erwinia-like' bacteria from animal sources and from other genera of the family Enterobacteriaceae were studied. The DNA base ratios of these bacteria fell mainly in the range $50-58 \% \mathrm{GC}$, consistent with their taxonomic placement in the family Enterobacteriaceae. Certain assemblages of erwinias could be grouped on the basis of GC content into clearly differentiated clusters, which, accidentally or otherwise, correlated neatly with certain existing nomenclatural groupings. It was not possible, from the GC values alone, to decide whether the present multiplicity of Erwinia species can be justified as separate species, or whether and how they might be combined into fewer species, or whether and how they might be demoted to some infraspecific level. Indeed, it is not clear whether the genus Erwinia, defined as it is solely on the basis of plant habitat, should be maintained or whether its species might not, just as rationally, be distributed throughout other genera of the family Enterobacteriaceae. Classifications of the genus Erwinia on over-all phenotypic similarity result in a small number of specific taxa which, on the basis of the present study, are seen to contain organisms of different GC contents. In the present state of the art, one can only be agnostic about the amount of such differences in GC content which corresponds to separate taxa or to a particular categoreal level (generic, specific, infraspecific) in the present hierarchical taxonomic scheme.

\section{INTRODUCTION}

The genus Erwinia was proposed originally by a committee (Winslow et al. 1917) with the candid admission that there was insufficient information for a more rational nomenclature: '.. the plant pathogens... seem to us perhaps to deserve distinct generic rank. They resemble the proteus group in their Gram-negative reaction, general growth characters, tendency toward slime formation, and generally negative indole reaction, but their fermentative powers are less vigorous and the exact biochemical changes they produce in carbohydrates are unknown. We suggest that all 
the peritrichate plant pathogens be for the present grouped under a new genus to be called Erwinia....'.

Erwin Frink Smith, the pioneer phytobacteriologist who was commemorated by this act, stated, quite resolutely (cf. Jones, 1939, p. 43): 'I have no sympathy with those who would...lump everything indiscriminately...the species I have mentioned ought not be put into one genus (Erwinia) simply because they are plant pathogens.' His advice went unheeded, and the causal agent of fire blight of pear-the first disease of plants proven to be caused by bacteria (Burrill, I882)-is today the type species (Erwinia amylovora) of the genus Erwinia. This impractical nomenclatural concept-in which taxa of phytopathogenic bacteria are segregated from other bacteria solely upon the basis of plant pathogenicity-has persisted to the present time. In Bergey's Manual (1957) the genus Erwinia is placed in the family Enterobacteriaceae, fenced off from its probable affinities not only by the nomenclatural barrier, but also by the insularity ensuing from the completely separate developments of plant and animal bacteriology.

Early in the twentieth century several bacterial soft-rot diseases of plants were studied and ascribed causally to several different bacterial 'species' which usually were named in accordance with the host plant from which they had been isolated. The genus Erwinia was sufficiently vague in concept to accommodate these soft-rot bacteria. Despite the early comparative studies (Jones, I90r; Harding \& Morse, I909; Jennison, 1923) which showed the essential similarity of most soft-rot bacteria to the one first described-now called Erwinia carotovora-a multiplicity of species names was used, with various definitions, but most frequently based on the curious 'new host-new species' notion (Starr, 1959; Stolp, Starr \& Baigent, 1965) However, it is clear that the usual soft-rot bacteria are as different from $E$. amylovora as, for example, Enterobacter cloacae is from Shigella dysenteriae. Non-phytopathogenic yellowpigmented bacteria are common on plants and some of these have been referred to the genus Erwinia (Billing \& Baker, 1963; Graham \& Hodgkiss, 1967). Finally, a group of yellow-pigmented bacteria from animals and humans is thought to be related to Erwinia (Muraschi, Friend \& Bolles, 1965; von Graevenitz \& Strouse, 1966; Whitcomb, Shapiro \& Richardson, 1966; Graham \& Hodgkiss, 1967; W. H. Ewing, personal communication; I. J. Slotnick, personal communication).

We have undertaken a comprehensive study of the genus Erwinia and related bacteria, including investigations on such aspects as nutrition (Starr \& Mandel, 1950); end-products of glucose dissimilation in E. amylovora (Sutton \& Starr, 1959), in $E$. carotovora (Kraght \& Starr, I952), and in other erwinias (in preparation); uronic acid catabolism (Kilgore \& Starr, 1959), pectolytic enzymes (Kraght \& Starr, I953; Starr \& Moran, I962; Nasuno \& Starr, I966; Moran, Nasuno \& Starr, 1968 $a, b$ ); pigment formation (Starr, Cosens \& Knackmuss, 1966); bacteriophage and bacteriocin relationships (in preparation); pathogenicity (Starr, 1947; Starr, Cardona \& Folsom, I95I ; in preparation). In this report, we present the DNA base ratios of representative cultures of Erwinia species and other enterobacteria. These data, together with the other information becoming available about these organisms, should eventually provide the basis for clarification of the taxonomic relationships of these phytopathogenic and other enterobacteria. 


\section{METHODS}

Organisms. The majority of the phytopathogenic bacteria were obtained from the International Collection of Phytopathogenic Bacteria (ICPB) maintained at the Department of Bacteriology, University of California, Davis. Many of these cultures are also accessioned in the National Collection of Plant Pathogenic Bacteria, Harpenden, Herts., England. The strains were chosen to be representative of the broadest possible range of geographical sources, host plants, and date of isolation. 'Erwinialike' bacteria isolated mainly from plant materials were provided by E. Billing (Billing \& Baker, 1963) and D. C. Graham (Graham \& Hodgkiss, 1967); those isolated from American white-tail deer, humans and leafhoppers were provided by T. F. Muraschi (Muraschi et al. 1965), I. J. Slotnick, R. F. Whitcomb (Whitcomb et al. 1966), W. H. Ewing, and A. von Graevenitz (von Graevenitz \& Strouse, 1966). Other enterobacteria came from the American Type Culture Collection (ATCC); these were selected to represent designated type or neotype cultures as far as these are available. Further details about the cultures are given in Tables I-3.

For clarity in this presentation, we will use the genus and species designations under which the culture was received. Several trivial names are used here in accordance with present practices of workers in this field: 'erwinia', meaning any organism referred to the genus Erwinia with no connotation regarding origin (plant, animal) or phytopathogenicity; 'Erwinia-like' bacteria, meaning definitely non-phytopathogenic erwinias from plants or animals; 'pectobacteria', meaning pectolytic (i.e. soft-rot) members of the genus Erwinia; 'true-Erwinia', meaning the non-pectolytic phytopathogens (E. amylovora and other non-pectolytic species) of the genus Erwinia; 'enterobacteria', meaning any member of the family Enterobacteriaceae, including all of the foregoing categories.

Deoxyribonucleic acid isolation and analysis. Lysates of bacteria for $\mathrm{CsCl}$ buoyant density ultracentrifugation were prepared from slant cultures, as previously described (Mandel, I966). Purified samples of DNA were prepared by the method of Marmur (1961) from organisms from 21 . cultures grown in Penassay broth (Difco) at $30^{\circ}$ with rotary agitation. These samples also were analysed in the ultracentrifuge for $\mathrm{CsCl}$ buoyant density, and their melting temperatures in $0.15 \mathrm{M}-\mathrm{NaCl}$ containing $0.015 \mathrm{M}$ sodium citrate at $\mathrm{pH} 7.0$ were determined by changes in extinction at $260 \mathrm{~m} \mu(\mathrm{nm}$.) (Mandel \& Marmur, 1968). The DNA base ratios were estimated from mean buoyant densities by means of the equation of Schildkraut, Marmur \& Doty (1962), and from the $T_{\mathrm{m}}$ by means of the equation of Marmur \& Doty (1962). Each DNA sample was analysed at least twice by $\mathrm{CsCl}$ buoyant density equilibrium centrifugation, and many were additionally examined by the extinction/temperature transition-technique. Following the usual convention, the DNA base ratios are expressed as molar percentages of guanine + cytosine ('GC content') in terms of the total base content.

\section{RESULTS AND DISCUSSION}

The GC contents of cultures ascribed to the genus Erwinia and to other genera of the Enterobacteriaceae are presented in Tables I-3. Table I contains the buoyant densities and calculated GC contents of the DNAs from the cultures of phytopathogenic erwinias, obtained by analysis of lysates. Table 2 presents the buoyant densities and 
Table I. Buoyant density in CsCl and GC content of DNA in lysates of cultures of Erwinia species and other phytopathogenic Enterobacteriaceae

\begin{tabular}{|c|c|c|c|}
\hline & $\begin{array}{l}\text { Strain } \\
\text { (ICPB) }\end{array}$ & $\begin{array}{l}\text { Mean density } \\
\left(\mathrm{g} . / \mathrm{cm} .^{3}\right)\end{array}$ & $\begin{array}{c}\text { GC } \\
(\mathrm{mole} \%)\end{array}$ \\
\hline E. amylovora & $\begin{array}{l}\text { EAII } \\
\text { EA I31 } \\
\text { EA I37 } \\
\text { EA I46 } \\
\text { EA I62 } \\
\text { EA I67 } \\
\text { EA I69 }\end{array}$ & $\begin{array}{l}I \cdot 7125 \\
I \cdot 713 \\
I \cdot 7127 \\
I \cdot 713 \\
I \cdot 713 \\
I \cdot 7125 \\
I \cdot 713\end{array}$ & $\begin{array}{l}53 \cdot 6 \\
54 \cdot 1 \\
53 \cdot 8 \\
54 \cdot I \\
54 \cdot I \\
53 \cdot 6 \\
54 \cdot I\end{array}$ \\
\hline E. ananas & $\begin{array}{l}\text { EA IOI } \\
\text { EA I33 } \\
\text { EA I80 } \\
\text { EA I8I }\end{array}$ & $\begin{array}{l}1 \cdot 7125 \\
1 \cdot 712 \\
1 \cdot 713 \\
1 \cdot 7125\end{array}$ & $\begin{array}{l}53 \cdot 6 \\
53 \cdot 1 \\
54 \cdot I \\
53 \cdot 6\end{array}$ \\
\hline E. aroideae & $\begin{array}{l}\text { EA I3 } \\
\text { EA I4 } \\
\text { EA I44 }\end{array}$ & $\begin{array}{l}1 \cdot 711 \\
I \cdot 712 \\
I \cdot 710\end{array}$ & $\begin{array}{l}52 \cdot 0 \\
53 \cdot 1 \\
51 \cdot 0\end{array}$ \\
\hline E. atroseptica & $\begin{array}{l}\text { EA I I } 2 \\
\text { EA I } 53\end{array}$ & $\begin{array}{l}I \cdot 7103 \\
I \cdot 712\end{array}$ & $\begin{array}{l}51 \cdot 3 \\
53 \cdot 1\end{array}$ \\
\hline E. carnegieana & $\begin{array}{l}\text { EC } 186 \\
\text { EC } 187 \\
\text { EC } 188 \\
\text { EC } 189 \\
\text { EC } 189 \\
\text { EC } 191 \\
\text { EC } 221 \\
\text { EC } 222 \\
\text { EC } 223\end{array}$ & $\begin{array}{l}I \cdot 710 \\
I \cdot 7098 \\
I \cdot 7107 \\
I \cdot 7103 \\
I \cdot 710 \\
I \cdot 710 \\
I \cdot 710 \\
I \cdot 7105 \\
I \cdot 710\end{array}$ & $\begin{array}{l}51 \cdot 0 \\
50 \cdot 8 \\
51 \cdot 7 \\
51 \cdot 3 \\
51 \cdot 0 \\
51 \cdot 0 \\
51 \cdot 0 \\
51 \cdot 5 \\
51 \cdot 0\end{array}$ \\
\hline E. carotovora & $\begin{array}{l}\text { EC IO5 } \\
\text { EC I3I } \\
\text { EC I } 38 \\
\text { EC } 150 \\
\text { EC I } 53 \\
\text { EC } 208\end{array}$ & $\begin{array}{l}I \cdot 710 \\
I \cdot 7115 \\
I \cdot 711 \\
I \cdot 7115 \\
I \cdot 7107 \\
I \cdot 7113\end{array}$ & $\begin{array}{l}51 \cdot 0 \\
52 \cdot 6 \\
52 \cdot 0 \\
52 \cdot 6 \\
51 \cdot 7 \\
52 \cdot 3\end{array}$ \\
\hline f.sp. parthenii & ECII5 & $I \cdot 7115$ & $52 \cdot 6$ \\
\hline E. cassavae & EC I I & $1 \cdot 714$ & $55^{\cdot 1}$ \\
\hline E. chrysanthemi & $\begin{array}{l}\text { EC16 } \\
\text { ECI75 } \\
\text { ECI83 } \\
\text { EC } 205\end{array}$ & $\begin{array}{l}I \cdot 714 \\
I \cdot 714 \\
I \cdot 7 I 5 \\
I \cdot 716\end{array}$ & $\begin{array}{l}55 \cdot \mathrm{I} \\
55 \cdot \mathrm{I} \\
56 \cdot \mathrm{I} \\
57 \cdot \mathrm{I}\end{array}$ \\
\hline Corn stalk-rot pathogen & $\begin{array}{l}\text { EM IO7 } \\
\text { EM IO8 } \\
\text { EM IO9 } \\
\text { EM I IO } \\
\text { EM I I I } \\
\text { EM I I I mut } \\
\text { EM I I } \\
\text { EC } 209 \\
\text { EC 210 } \\
\text { EC 210 mut }\end{array}$ & $\begin{array}{l}I \cdot 7125 \\
I \cdot 713 \\
I \cdot 712 \\
I \cdot 7125 \\
I \cdot 7125 \\
I \cdot 7125 \\
I \cdot 713 \\
I \cdot 7125 \\
I \cdot 7125 \\
I \cdot 7125\end{array}$ & $\begin{array}{l}53 \cdot 6 \\
54 \cdot 1 \\
53 \cdot 1 \\
53 \cdot 6 \\
53 \cdot 6 \\
53 \cdot 6 \\
54 \cdot 1 \\
53 \cdot 6 \\
53 \cdot 6 \\
53 \cdot 6\end{array}$ \\
\hline E. cypripedii & $\begin{array}{l}\text { EC } 155 \\
\text { EC } 160\end{array}$ & $\begin{array}{l}I \cdot 713 \\
I \cdot 7135\end{array}$ & $\begin{array}{l}54 \cdot 1 \\
54 \cdot 6\end{array}$ \\
\hline E. cytolytica & EC 207 & $1 \cdot 715$ & $56 \cdot I$ \\
\hline E. dieffenbachiae & $\begin{array}{l}\text { ED IO2 } \\
\text { ED IO3 }\end{array}$ & $\begin{array}{l}I \cdot 714 \\
I \cdot 715\end{array}$ & $\begin{array}{l}55 \cdot 1 \\
56 \cdot 1\end{array}$ \\
\hline
\end{tabular}


Table I (cont.)

\begin{tabular}{|c|c|c|c|}
\hline & $\begin{array}{l}\text { Strain } \\
\text { (ICPB) }\end{array}$ & $\begin{array}{l}\text { Mean density } \\
\left(\mathrm{g} . / \mathrm{cm} .^{3}\right)\end{array}$ & $\begin{array}{c}\text { GC } \\
(\text { mole \%) }\end{array}$ \\
\hline E. dissolvens & $\begin{array}{l}\text { ED I05 } \\
\text { ED IO6 } \\
\text { ED I09 } \\
\text { ED IIO } \\
\text { ED III } \\
\text { ED II } \\
\text { ED II3 } \\
\text { ED II4 }\end{array}$ & $\begin{array}{l}I \cdot 7125 \\
I \cdot 716 \\
1 \cdot 7165 \\
I \cdot 714 \\
I \cdot 7147 \\
I \cdot 716 \\
I \cdot 7155 \\
I \cdot 716\end{array}$ & $\begin{array}{l}53 \cdot 6 \\
57 \cdot 1 \\
57 \cdot 6 \\
55 \cdot 1 \\
55 \cdot 8 \\
57 \cdot 1 \\
56 \cdot 6 \\
57 \cdot 1\end{array}$ \\
\hline E. mangiferae & EM IOI & $I \cdot 7135$ & $54 \cdot 6$ \\
\hline E. milletiae & $\begin{array}{l}\text { EM IO2 } \\
\text { EMII3 } \\
\text { EMII4 }\end{array}$ & $\begin{array}{l}I \cdot 7 I 44 \\
I \cdot 7 I 3 \\
I \cdot 7 I 4\end{array}$ & $\begin{array}{l}55 \cdot I \\
54 \cdot I \\
55^{\cdot} \cdot I\end{array}$ \\
\hline E. nigrifluens & EN 104 & $1 \cdot 715$ & $56 \cdot I$ \\
\hline E. nimipressuralis & EN I & $1 \cdot 714$ & $55 \cdot \mathrm{I}$ \\
\hline E. oleraceae & EO I & I.7095 & $50 \cdot 5$ \\
\hline E. quercina & $\begin{array}{l}\text { EQ IOI } \\
\text { EQ IO2 }\end{array}$ & $\begin{array}{l}I \cdot 714 \\
1 \cdot 7135\end{array}$ & $\begin{array}{l}55 \cdot 1 \\
54 \cdot 6\end{array}$ \\
\hline E. rhapontici & $\begin{array}{l}\text { ER I } \\
\text { ER IO2 } \\
\text { ER IO6 }\end{array}$ & $\begin{array}{l}I \cdot 710 \\
I \cdot 712 \\
I \cdot 712\end{array}$ & $\begin{array}{l}5 I \cdot 0 \\
53 \cdot I \\
53 \cdot I\end{array}$ \\
\hline E. rubrifaciens & $\begin{array}{l}\text { ER I03 } \\
\text { ER I04 } \\
\text { ER I05 }\end{array}$ & $\begin{array}{l}I \cdot 711 \\
I \cdot 7115 \\
I \cdot 7115\end{array}$ & $\begin{array}{l}52 \cdot 0 \\
52 \cdot 6 \\
52 \cdot 6\end{array}$ \\
\hline E. salicis & $\begin{array}{l}\text { ES } 4 \\
\text { ES } 102\end{array}$ & $\begin{array}{l}I \cdot 7103 \\
I \cdot 7105\end{array}$ & $\begin{array}{l}5 I \cdot 3 \\
5 I \cdot 5\end{array}$ \\
\hline E. solanisapra & ES IOI & $1 \cdot 710$ & $51 \cdot 0$ \\
\hline E. tracheiphila & $\begin{array}{l}\text { ET } 5 \\
\text { ET } 102 \\
\text { ET } 106\end{array}$ & $\begin{array}{l}I \cdot 7 \mathrm{II} \\
\mathrm{I} \cdot 7095 \\
\mathrm{I} \cdot 709\end{array}$ & $\begin{array}{l}52 \cdot 0 \\
50 \cdot 5 \\
50 \cdot 0\end{array}$ \\
\hline $\begin{array}{l}\text { 'Aerobacter aerogenes' } \\
\text { (banana strain) }\end{array}$ & $315 I$ & $1 \cdot 715$ & $56 \cdot 1$ \\
\hline 'Bacterium stewartii' & $\begin{array}{l}\text { SSII } \\
\text { SSIO2 }\end{array}$ & $\begin{array}{l}1 \cdot 714 \\
I \cdot 7135\end{array}$ & $\begin{array}{l}55 \cdot I \\
54 \cdot 6\end{array}$ \\
\hline 'Xanthomonas uredovorus' & $\begin{array}{l}\text { XU I03 } \\
\text { XU I04 }\end{array}$ & $\begin{array}{l}I \cdot 712 \\
I \cdot 712\end{array}$ & $\begin{array}{l}53 \cdot I \\
53 \cdot I\end{array}$ \\
\hline
\end{tabular}

calculated GC contents of the DNAs in the lysates of the cultures of non-phytopathogenic Erwinia-like bacteria. Table 3 presents comparative calculations of the GC contents determined both in $\mathrm{CsCl}$ and by thermal denaturation on purified samples of DNA extracted from representative Erwinia cultures and reference cultures of other enterobacteria. Table 4 summarizes tests of statistical significance of pairwise differences in densities between selected sets of Erwinia species; there is no necessity to equate such statistically significant differences with taxonomic significance.

The data have been applied to several purposes. Where the cultures have already been assigned to a specific taxon, the uniformity of the taxon has been tested with regard to the uniformity of GC contents. Various proposals for combining existing taxa have been examined in the light of the GC data. The wisdom of referring the non-phytopathogenic Erwinia-like bacteria to the family Enterobacteriaceae has been evaluated in terms of the GC values. The homogeneity of the family Enterobacteria- 
ceae, with the various erwinias included, is scrutinized. The rationality of maintaining a genus Erwinia is considered. Since a rather large number of DNAs were examined by both buoyant density and transition temperature techniques, comparisons are made between the two methods (Table 3).

Table 2. Buoyant density in $\mathrm{CsCl}$ and $G C$ content of $D N A$ in lysates of non-phytopathogenic 'Erwinia-like' bacteria

\begin{tabular}{|c|c|c|c|}
\hline Source or Name & $\begin{array}{l}\text { Strain } \\
\text { (ICPB) }\end{array}$ & $\begin{array}{l}\text { Mean density } \\
\left(\mathrm{g} . / \mathrm{cm} .^{3}\right)\end{array}$ & $\underset{(\text { mole \%) }}{\text { GC }}$ \\
\hline Plant materials (Billing) & $\begin{array}{l}2650-65 \mathrm{I} \\
2650-653 \\
2650-656 \\
2650-658 \\
2650-66 \mathrm{I} \\
2650-662\end{array}$ & $\begin{array}{l}I \cdot 714 \\
I \cdot 713 \\
I \cdot 7145 \\
I \cdot 712 \\
I \cdot 714 \\
I \cdot 7135\end{array}$ & $\begin{array}{l}55 \cdot \mathrm{I} \\
54 \cdot \mathrm{I} \\
55 \cdot 6 \\
53 \cdot \mathrm{I} \\
55 \cdot \mathrm{I} \\
54 \cdot 6\end{array}$ \\
\hline Erwinia lathyri & $\begin{array}{l}\text { EL IOI B } \\
\text { EL IO2 } \\
\text { EL 103 } \\
\text { EL IO4 } \\
\text { EL I05 } \\
\text { EL IO6 } \\
\text { EL IO7 }\end{array}$ & $\begin{array}{l}I \cdot 714 \\
I \cdot 714 \\
I \cdot 714 \\
1 \cdot 7115 \\
I \cdot 712 \\
I \cdot 7165 \\
I \cdot 713\end{array}$ & $\begin{array}{l}55 \cdot \mathrm{I} \\
55 \cdot \mathrm{I} \\
55 \cdot \mathrm{I} \\
52 \cdot 6 \\
53 \cdot \mathrm{I} \\
57 \cdot 7 \\
54 \cdot \mathrm{I}\end{array}$ \\
\hline Erwinia herbicola & $\begin{array}{l}2553 \\
2554 \\
3161 \\
3162 \\
3163\end{array}$ & $\begin{array}{r}1 \cdot 714 \\
\mathrm{I} \cdot 713 \\
\mathrm{I} \cdot 714 \\
\mathrm{I} \cdot 7 \mathrm{I} \\
\mathrm{I} \cdot 714\end{array}$ & $\begin{array}{l}55 \cdot \mathrm{I} \\
54 \cdot \mathrm{I} \\
55 \cdot \mathrm{I} \\
53 \cdot \mathrm{I} \\
55 \cdot \mathrm{I}\end{array}$ \\
\hline 'Xanthomonas trifolii' & $\begin{array}{l}\text { XT IO9 } \\
\text { XTIIO }\end{array}$ & $\begin{array}{l}I \cdot 7145 \\
I \cdot 71455\end{array}$ & $\begin{array}{l}55^{\cdot} 6 \\
55^{\cdot} \cdot\end{array}$ \\
\hline Deer (Muraschi) & $\begin{array}{l}2948 \\
2949 \\
2950\end{array}$ & $\begin{array}{l}I \cdot 7 I 449 \\
I \cdot 7 I 4 \\
I \cdot 7 I 5\end{array}$ & $\begin{array}{l}56 \cdot 0 \\
55 \cdot I \\
56 \cdot I\end{array}$ \\
\hline Leafhopper (Whitcomb) & $\begin{array}{l}2973-\mathrm{L} \\
2973-\mathrm{S}\end{array}$ & $\begin{array}{l}I \cdot 7 I I 5 \\
I \cdot 7125\end{array}$ & $\begin{array}{l}52 \cdot 6 \\
53 \cdot 6\end{array}$ \\
\hline Man (Slotnick) & $\begin{array}{l}2858-\mathrm{A} \\
2858-\mathrm{B}\end{array}$ & $\begin{array}{l}1 \cdot 7145 \\
I \cdot 714\end{array}$ & $\begin{array}{l}55 \cdot 6 \\
55 \cdot I\end{array}$ \\
\hline Man (Muraschi) & $\begin{array}{l}2953 \\
2956\end{array}$ & $\begin{array}{l}I \cdot 7155 \\
I \cdot 713\end{array}$ & $\begin{array}{l}56 \cdot 6 \\
54 \cdot 1\end{array}$ \\
\hline Man (von Graevenitz) & $\begin{array}{l}2984 \\
2986 \\
2987\end{array}$ & $\begin{array}{l}I \cdot 719 \\
I \cdot 7185 \\
I \cdot 715\end{array}$ & $\begin{array}{l}60 \cdot 2 \\
59 \cdot 7 \\
56 \cdot 1\end{array}$ \\
\hline Man (Ewing) & $\begin{array}{l}2992 \\
3080\end{array}$ & $\begin{array}{l}I \cdot 714 \\
I \cdot 7145\end{array}$ & $\begin{array}{l}55 \cdot I \\
55 \cdot 6\end{array}$ \\
\hline 'Bacterium typhi flavum' & $\begin{array}{l}3164 \\
3165\end{array}$ & $\begin{array}{l}1 \cdot 714 \\
1 \cdot 714\end{array}$ & $\begin{array}{l}55 \cdot \mathrm{I} \\
55 \cdot \mathrm{I}\end{array}$ \\
\hline
\end{tabular}

The proposalof Waldee (1945) to separate the soft-rot bacteria (as a genus Pectobacterium) from the rest of the genus Erwinia is neither supported nor confirmed by the GC data. The assortments within these two groups, the pectobacteria and the true-Erwinia, as reviewed by Graham (1964), can now be assessed in terms of GC contents. Erwinia amylovora $(53.9 \%$ GC) could accommodate $E$. cassavae and $E$. mangiferae. The DNAs of E. salicis and E. tracheiphila, on the other hand, are $4 \%$ to $5 \%$ lower in GC content than those of E. amylovora and its relatives. In addition, 
the yellow-pigmented erwinias (Dye, 1963; Graham \& Hodgkiss, 1967)—'Xanthomonas uredovorus', E. ananas, E. lathyri, and 'Bacterium stewartii'-as well as many of the yellow Erwinia-like bacteria, have GC contents not unlike the $E$. amylovora group and lower than the Pseudomonadaceae genera in which some of them had earlier

Table 3. Melting temperatures, buoyant densities in $\mathrm{CsCl}$, and calculated $\mathrm{GC}$ contents of purified DNA samples from representative Erwinia and other Enterobacteriaceae

\begin{tabular}{|c|c|c|c|c|c|}
\hline Name & Strain & $\begin{array}{c}T_{\mathrm{m}} \\
\left({ }^{\circ} \mathrm{C}\right)\end{array}$ & $\begin{array}{c}\text { GC } \\
(\text { mole } \%)\end{array}$ & $\begin{array}{c}\text { Buoyant } \\
\text { density } \\
\left(\mathrm{g} . / \mathrm{cm} .^{3}\right)\end{array}$ & $\begin{array}{c}\mathrm{GC} \\
(\mathrm{mole} \%)\end{array}$ \\
\hline Erwinia amylovora & ICPB EA I I & $9 \mathrm{I} \cdot \mathrm{O}$ & $53 \cdot 0$ & $I \cdot 7125$ & $53 \cdot 6$ \\
\hline E. aroideae & ICPB EA I 4 & $91 \cdot 5$ & 54.0 & $1 \cdot 712$ & $53 \cdot I$ \\
\hline E. carotovora & ICPB EC I 38 & $9 \mathrm{I} \cdot 2$ & $53 \cdot 4$ & I 7 I I & $52 \cdot 0$ \\
\hline E. chrysanthemi & ICPB EC I 6 & $92 \cdot I$ & $55 \cdot 6$ & $I \cdot 7 I 4$ & $55^{\cdot} \mathrm{I}$ \\
\hline E. dissolvens & ICPB ED 106 & $92 \cdot 7$ & $57 \cdot \mathrm{I}$ & $I \cdot 716$ & $57 \cdot 1$ \\
\hline E. lathyri & ICPB EL 103 & $92 \cdot 2$ & $55 \cdot 9$ & $I \cdot 7 I 4$ & $55 \cdot I$ \\
\hline E. milletiae & ICPB EM 102 & $92 \cdot 0$ & $55 \cdot 4$ & $I \cdot 7 I 4$ & $55 \cdot I$ \\
\hline E. nigrifluens & ICPB EN IO4 & $92 \cdot 5$ & $56 \cdot 6$ & $I \cdot 715$ & $56 \cdot I$ \\
\hline E. nimipressuralis & ICPB EN I & 92.0 & $55 \cdot 4$ & $I \cdot 714$ & $55 \cdot 1$ \\
\hline E. oleraceae & ICPB EO I & $90 \cdot 4$ & $5 \mathrm{I} \cdot 5$ & $I \cdot 7095$ & $50 \cdot 5$ \\
\hline E. rhapontici & ICPB ER I & $90 \cdot 2$ & $5 I \cdot I$ & $1 \cdot 710$ & $5 I \cdot 0$ \\
\hline E. salicis & ICPB ES 4 & $90 \cdot 8$ & $52 \cdot 5$ & $1 \cdot 7103$ & $51 \cdot 3$ \\
\hline E. tracheiphila & ICPB ET 106 & 90.9 & $52 \cdot 7$ & $1 \cdot 709$ & $50 \cdot 0$ \\
\hline Escherichia aurescens & ATCC I 2814 & $90 \cdot 3$ & $5 I \cdot 3$ & $1 \cdot 710$ & $51 \cdot 0$ \\
\hline E. coli & K-I 2 Hfr G 6 & $90 \cdot 4$ & $51 \cdot 5$ & $1 \cdot 710$ & $51 \cdot 0$ \\
\hline E. coli & ATCC II 1775 & $90 \cdot 5$ & $51 \cdot 7$ & $1 \cdot 710$ & $51 \cdot 0$ \\
\hline E. coli & ATCC I 28I4 & $90 \cdot 3$ & $51 \cdot 3$ & $1 \cdot 710$ & $51 \cdot 0$ \\
\hline Enterobacter aerogenes & ATCC 13048 & $91 \cdot 8$ & $54 \%$ & $1 \cdot 712$ & $53 \cdot 1$ \\
\hline E. aerogenes & ATCC 13882 & $92 \cdot I$ & $55 \cdot 6$ & $I \cdot 715$ & $56 \cdot I$ \\
\hline E. alvei & ATCC 23280 & $89 \cdot 2$ & $48 \cdot 7$ & $x \cdot 707$ & $48 \cdot 0$ \\
\hline E. cloacae & ATCC 13047 & $92 \cdot 0$ & $55 \cdot 4$ & $1 \cdot 7135$ & $54 \cdot 6$ \\
\hline E. lipolyticus & ATCC I 4460 & $9 I \cdot 4$ & 53.9 & $1 \cdot 7115$ & $52 \cdot 6$ \\
\hline Klebsiella pneumoniae & ATCC 13883 & $92 \cdot 9$ & $57 \cdot 6$ & $1 \cdot 7123$ & $53 \cdot 4$ \\
\hline $\begin{array}{l}\text { K. cdwardsii subsp. } \\
\text { edwardsii }\end{array}$ & ATCC 13886 & $92 \cdot 5$ & $56 \cdot 5$ & I 7155 & $56 \cdot 6$ \\
\hline $\begin{array}{l}\text { K. edwardsii subsp. } \\
\text { atlantae }\end{array}$ & ATCC 13887 & $92 \cdot 6$ & $56 \cdot 9$ & I. 715 & $56 \cdot I$ \\
\hline K. rhinoscleromatis & ATCC 13884 & $92 \cdot 7$ & $57 \cdot I$ & $I \cdot 7 I 45$ & $55^{\circ} 6$ \\
\hline Citrobacter freundii & ATCC 8090 & $90 \cdot 9$ & $52 \cdot 7$ & $1 \cdot 7115$ & $52 \cdot 6$ \\
\hline
\end{tabular}

Table 4. Tests of statistical significance of pairwise differences in bouyant densities of the DNAs from selected sets of Erwinia species

\begin{tabular}{|c|c|c|c|c|c|}
\hline & $x \pm \sigma$ & $n$ & $t$ & D.F. & $\begin{array}{l}\rho \text { (sign } \\
\text { ignored) }\end{array}$ \\
\hline $\begin{array}{l}\text { E. carnegieana } \\
\text { E. carotovora }\end{array}$ & $\begin{array}{l}I \cdot 7100 \pm 0.0008 \\
\text { I. } 7 \text { IIII } \pm 0.0009\end{array}$ & $\left.\begin{array}{l}22 \\
17\end{array}\right\}$ & $3 \cdot 78$ & 37 & 0.001 \\
\hline $\begin{array}{l}\text { E. amylovora } \\
\text { E. salicis }\end{array}$ & $\begin{array}{l}I \cdot 7128 \pm 0.0007 \\
I \cdot 7105 \pm 0.0006\end{array}$ & $\left.\begin{array}{r}16 \\
4\end{array}\right\}$ & $6 \cdot 6$ & 18 & 0.001 \\
\hline $\begin{array}{l}\text { E. chrysanthemi } \\
\text { 'Corn stalk rotters' }\end{array}$ & $\begin{array}{l}1 \cdot 7148 \pm 0.0009 \\
1 \cdot 7126 \pm 0.0005\end{array}$ & $\left.\begin{array}{r}8 \\
23\end{array}\right\}$ & $8 \cdot 45$ & 29 & $>0.001$ \\
\hline $\begin{array}{l}\text { E. amylovora } \\
\text { E. ananas }\end{array}$ & $\begin{array}{l}1 \cdot 7128 \pm 0.0007 \\
I \cdot 7125 \pm 0.0005\end{array}$ & $\left.\begin{array}{r}16 \\
8\end{array}\right\}$ & 0.034 & 22 & $<0.5$ \\
\hline $\begin{array}{l}\text { E. carotovora } \\
\text { E. aroideae }\end{array}$ & $\begin{array}{l}\text { I.7IIII } \pm 0.0009 \\
\text { I.7IIIO } \pm 0.0011\end{array}$ & $\left.\begin{array}{r}17 \\
6\end{array}\right\}$ & 0.874 & 2 I & 0.4 \\
\hline
\end{tabular}


been included (see De Ley, 1968, for an independent study on this point). The Erwinialike organisms isolated from American white-tail deer (Muraschi), from man (Ewing, Slotnick, Muraschi, and one of the three strains sent by von Graevenitz), from leafhoppers (Whitcomb), and the non-phytopathogenic plant Erwinia-like organisms received from Billing and from Graham have GC contents which are also similar to the E. amylovora range, and these are all similar to the data for Klebsiella and certain Enterobacter cultures. Two of the three erwinia strains isolated from humans by von Graevenitz \& Strouse (I966) have higher GC contents (60.2\%, 59.7\% GC) than any other Enterobacteriaceae cultures examined in this study.

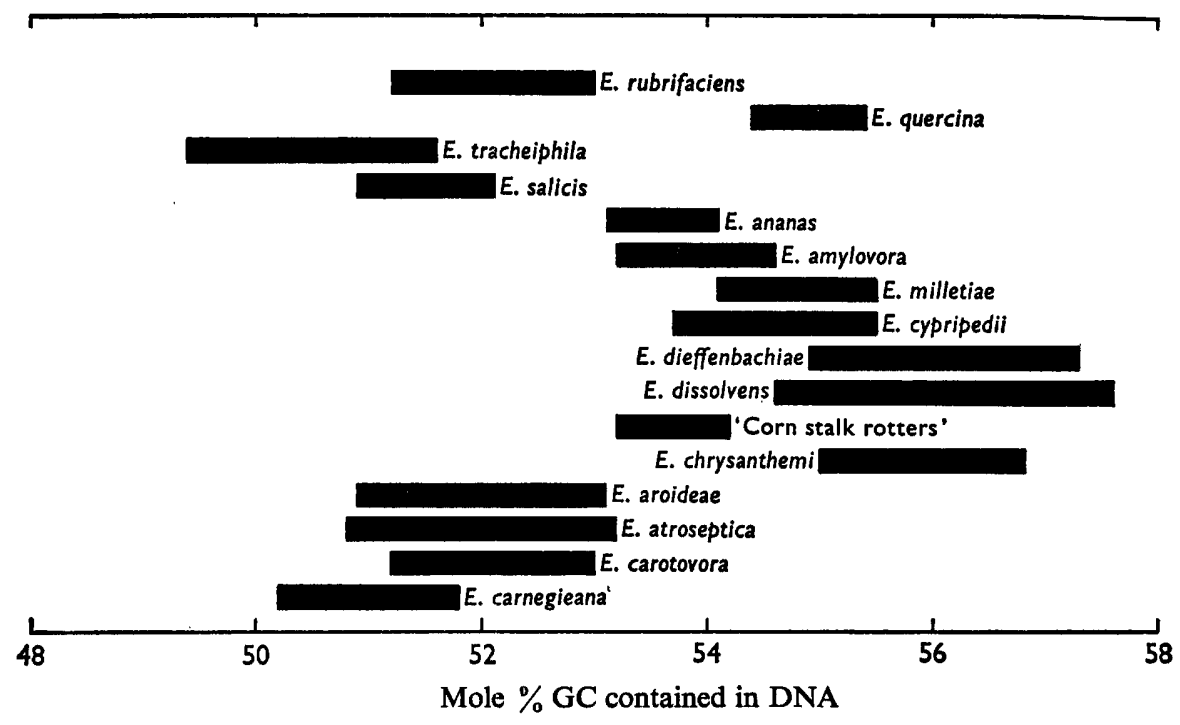

Fig. I. The GC contents of the DNAs of representative Erwinia species. The values plotted are means \pm one standard deviation.

Turning to the soft-rot erwinias, the pectobacteria, Erwinia carotovora $(52 \cdot 1 \%$ $\mathrm{GC}$ ) does not display any tendency to discrete GC contents for its relatives $E$. aroideae, $E$. solanisapra, E. oleraceae, and E. atroseptica, thus justifying the current practice of lumping these into one species. Erwinia chrysanthemi is characterized by $56 \%$ GC and, on this basis, cannot be considered to be identical with $E$. carotovora $(52 \cdot 1 \%$ GC). The several organisms rotting the stalks of corn plants (A. Kelman, unpublished) are significantly different (i.e. statistically so; Table 4) in their GC contents $(53.7 \%$ GC) from the E. carotovora ( $52 \cdot \mathrm{I} \% \mathrm{GC})$, E. chrysanthemi $(56 \% \mathrm{GC})$, and $E$. dissolvens $(56.3 \% \mathrm{GC})$ cultures; they might form a taxon separate from these three, but whether at the specific or infraspecific level remains to be decided on other grounds. Similarly, the representatives of E. carnegieana ( $5 \mathrm{I} \cdot \mathrm{I} \% \mathrm{GC})$ are distinct in their $\mathrm{GC}$ contents, as compared to the other soft-rot erwinias, and might be viewed as a separate taxon; here again, the hierarchical level cannot be decided from these data alone.

The other named strains which have been examined in this study fall into the range of 50-58\% GC values, typical of the other members of the family Enterobacteriaceae (Marmur, Falkow \& Mandel, I963; Hill, I966; Rosypal \& Rosypalová, I966). The 
extremes are reached with Enterobacter alvei ATCC $23280(48 \cdot 0 \% \mathrm{GC})$ and the aforementioned human erwinia strain from von Graevenitz, ICPB $2984(60 \cdot 2 \% \mathrm{GC})$.

Based on a study of over-all phenotypic similarity, Martinec \& Kocur (1963) have suggested that all the species presently in the genus Erwinia might be reduced to two species: $E$. carotovora and E. amylovora (and var. salicis). In a similar study with over 200 cultures and using some 80 traits, Dye (1968) has recommended reduction to five species: E. amylovora (and var. salicis, var. tracheiphila, var. nigrifluens, var. quercina), E. herbicola (and var. ananas), E. uredovorus, E. stewartii, E. carotovora (and var. atrospetica, var. chrysanthemi, var. cypripedii, var. rhapontici). Since following these recommendations would lead to taxa (specific and infraspecific) which the present DNA data show would be composed of organisms with statistically different GC contents (Table 4), we are at a loss to decide on the wisdom of these suggestions. In support of this view, the computer sorting of Lockhart \& Koenig (1965) and Krieg \& Lockhart (I966) shows no intensification of sorting when the S-values which they report for Erwinia species are plotted versus our data on their GC contents. Indeed, the range of link-sorting is less than the range of GC values throughout the family Enterobacteriaceae; hence, the traits used in the generation of these computer analyses are probably of little value in discriminating taxa at the generic level and below. The data may, of course, also mean that a species which is based on high over-all phenotypic similarity can include individual specimens with genetic make-ups varying over a range corresponding to several per cent in GC content.

We are not now prepared to equate discrete differences in GC contents with discrete taxa (see Rosypal \& Rosypalová, I966). In the present state of the art, who is to say what particular amount of difference in DNA base ratios or in DNA homologies justifies separating one taxon from another? We can not now be other than agnostic about the amount of such differences which would be required to place taxa in any particular position in the hierarchial taxonomic categories. All we can now say with assurance is that certain assemblages of erwinias can be grouped on the basis of GC contents into clearly differentiated clusters, and that these clusters-accidentally or otherwise-correlate neatly with certain existing nomenclatural groupings.

There is no compelling evidence from the GC data that the Erwinia group, either whole or in parts, comprises a taxon or taxa separable at the generic level from the other genera of the Enterobacteriaceae. Indeed, from Fig. I it will be seen that a case could be made for distributing the erwinias throughout the other genera of the family Enterobacteriaceae. Broadly based comparative studies now in progress are designed to clarify this matter; hence, no nomenclatural recommendations will be made here.

Supported in part by research grant AI-08426 from the National Institute of Allergy and Infectious Diseases, United States Public Health Service, and by a Fellowship from the John Simon Guggenheim Memorial Foundation. We gratefully acknowledge expert technical assistance by Gladys Cosens, Janet A. Bergendahl, D. L. Sackett, and D. Mandell.

\section{REFERENCES}

BILIING, E. \& BAKER, L. A. E. (1963). Characteristics of Erwinia-like organisms found in plant material. J. appl. Bact. 26, 58.

Bergey's Manual of Determinative Bacteriology. (1957). 7th ed. Ed. by R. S. Breed, E. G. D. Murray and N. R. Smith. Baltimore: Williams and Wilkins Co. 
BURRILL, T. J. (1882). The bacteria: an account of their nature and effects, together with a systematic description of the species. Illinois Ind. Univ., Report of the Board of Trustees II, 93.

De LEY, J. (1968). DNA base composition of yellow Erwinia strains. Antonie van Leeuwenhoek 34, 257.

DYE, D. W. (1963). The taxonomic position of Xanthomonas uredovorus Pon et al. 1954. N.Z. Jl Sci. 6, 146 .

Dye, D. W. (1968). A taxonomic study of the genus Erwinia. Ist. Int. Congr. Pl. Path. (London, July I968), Abstracts of Papers, 5 I.

Graevenitz, A. von, \& Strouse, A. (1966). Isolation of Erwinia spp. from human sources. Antonie van Leeuwenhoek 32, 429.

Graham, D. C. (1964). Taxonomy of the soft-rot coliform bacteria. A. Rev. Phytopath. 2, i3.

GraHAM, D. C. \& HoDGKISS, W. (1967). Identity of Gram negative, yellow pigmented, fermentative bacteria isolated from plants and animals. J. appl. Bact. 30, 175 .

HARDING, H. A. \& MoRSE, W. J. (1909). The bacterial soft rots of certain vegetables. Tech. Bull. N.Y. St. agric. Exp. Stn. II, 251.

HILL, L. R. (1966). An index to deoxyribonucleic acid base compositions of bacterial species. J. gen. Microbiol. 44, 419.

Jennison, H. M. (1923). Potato blackleg with special reference to the etiological agent. Ann. Mo. bot. Gdn. 10, I.

JONES, L. R. (190I). A soft rot of carrot and other vegetables caused by Bacillus carotovorus. Rep. Vermont. agric. Exp. Stn 13, 299.

JoNes, L. R. (1939). Biographical memoir of Erwin Frink Smith I854-1927. Biogr. Mem. natn. Acad. Sci. 2I, I.

KILGORE, W. W. \& STARR, M. P. (1959). Catabolism of galacturonic and glucuronic acids by Erwinia carotovora. J. biol. Chem. 234, 2227.

Kraght, A. J. \& Starr, M. P. (1952). Fermentation of galacturonic acid and glucose by a strain of Erwinia carotovora. J. Bact. 64, 259.

Kraght, A. J. \& Starr, M. P. (1953). Pectic enzymes of Erwinia carotovora. Archs. Biochem. Biophys. 42, 271.

KRIEG, R. E. \& LOCKHART, W. R. (1966). Classification of enterobacteria based on overall similarity. J. Bact. 42, I275.

LOCKHART, W. R. \& KoENIG, K. (1965). Use of secondary data in numerical taxonomy of the genus Erwinia. J. Bact. 90, I638.

Mandel, M. (1966). Deoxyribonucleic acid base composition in the genus Pseudomonas. $J$ gen. Microbiol. 43, 273.

MANDEL, M. \& MARMUR, J. (I968). Use of ultraviolet absorbance-temperature profile for determining the guanine plus cytosine content of DNA. Meth. Enzym. 12, 195.

MARMUR, J. (I96I). A procedure for the isolation of deoxyribonucleic acid from micro-organisms. J. molec. Biol. 3, 208.

MarmuR, J. \& Doty, P. (1962). Determination of the base composition of dexoyribonucleic acid from its thermal denaturation temperature. J. molec. Biol. 5, 109.

Marmur, J., Falkow, S. \& Mandel, M. (1963). New approaches to bacterial taxonomy. A. Rev. Microbiol. 17, 329.

Martinec, T. \& Kocur, M. (1963). A taxonomic study of the genus Erwinia. Folia. Publ. Fac. Nat. Sci. J. E. Purkynye Univ. (Biologia), 4, I.

Moran, F., NAsuno, S. \& StarR, M. P. (1968a). Extracellular and intracellular polygalacturonic acid trans-eliminases of Erwinia carotovora. Archs Biochem. Biophys. 123, 298.

Moran, F., Nasuno, S. \& StarR, M. P. (1968b). Oligogalacturonide trans-eliminase of Erwinia carotovora. Archs Biochem. Biophys. 125, 734.

MurasChI, T. F., FrIEND, M. \& Bolles, D. (1965). Erwinia-like microorganisms isolated from animal and human hosts. Appl. Microbiol. 13, I28.

NAsuno, S. \& Starr, M. P. (I966). Polygalacturonase of Erwinia carotovora. J. biol. Chem. 24I, 5298.

Rosypal, S. \& Rosypalová A. (I966). Genetic, phylogenetic and taxonomic relationships among bacteria as determined by their deoxyribonucleic acid base composition. Folia. Publ. Fac. Nat. Sci. J. E. Purkynye Univ. (Biologia) 7, I. 
Schildkraut, C. L., Marmur, J. \& Doty, P. (I962). Determination of the base composition of deoxyribonucleic acid from its buoyant density in CsCl. J. molec. Biol. 4, 430.

StaRr, M. P. (1947). The cause of bacterial root and stem disease of guayule. Phytopathology 37, 291.

StarR, M. P. (1959). Bacteria as plant pathogens. A. Rev. Microbiol. 13, 2 I I.

STARR, M. P. \& MANDEL, M. (I950). The nutrition of phytopathogenic bacteria. IV. Minimal nutritive requirements of the genus Erwinia. J. Bact. 6o, 669.

StarR, M. P. \& MORAN, F. (1962). Eliminative split of pectic substances by phytopathogenic soft-rot bacteria. Science, N.Y. 135, 920.

Starr, M. P., Cardona, C. \& Folsom, D. (195I). Bacterial fire blight of the raspberry. Phytopathology 4r, 915.

Starr, M. P., Cosens, G. \& KNackmuss, H.-J. (1966). Formation of the blue pigment indigoidine by phytopathogenic Erwinia. Appl. Microbiol. 14, 870.

StolP, H. StarR, M. P. \& Baigent, N. L. (1965). Problems in speciation of phytopathogenic pseudomonads and xanthomonads. A. Rev. Phytopath. 3, 231.

SUtTon, D. D. \& STARR, M. P. (I959). Anaerobic dissimilation of glucose by Erwinia amylovora. J. Bact. 78, 427.

WALDEE, E. L. (I945). Comparative studies of some peritrichous phytopathogenic bacteria. Iowa St. Coll. J. Sci. $\mathbf{1 9}, 435$.

Whitcomb, R. F., Shapiro, M. \& Richardson, J. (1966). An Erwinia-like bacterium pathogenic to leafhoppers. J. Invertebr. Path. 8, 299.

Winslow, C. E. A., Broadhurst, J., Buchanan, R. E., Krumwiede, C. Jun., Rogers, L. A. \& SмIтH, G. H. (I917). The families and genera of the bacteria. Preliminary report of the committee of the Society of American Bacteriologists on characterization and classification of bacterial types. J. Bact. 2, 505. 